\title{
Cerebral blood flow during treatment for pulmonary hypertension
}

\author{
Satoshi Kusuda, Norio Shishida, Nobuhiro Miyagi, Madoka Hirabayashi, Tae-Jang Kim
}

\begin{abstract}
Aim-To determine if the haemodynamics of systemic and cerebral circulation are changed during treatment for persistent pulmonary hypertension of the newborn (PPHN).

Methods-Fifteen term newborn piglets with hypoxia induced pulmonary hypertension were randomly assigned either tolazoline infusion $(\mathbf{T z})$, hyperventilation alkalosis (HAT), and inhaled nitric oxide (iNO). Mean pulmonary arterial pressure (PAP), mean systemic arterial pressure (SAP), and cerebral blood flow volume (CBF) were measured.

Results-During hypoxic breathing, PAP increased significantly in all groups. After treatment PAP decreased significantly in all groups, but no significant difference was observed between groups. SAP decreased significantly only in the $\mathbf{T z}$ group, and $C B F$ reduced significantly only in the HAT group. On the other hand, iNO did not change SAP or CBF.

Conclusion-Inhaled NO might be ideal for the resolution of pulmonary hypertension.

(Arch Dis Child Fetal Neonatal Ed 1999;80:F30-F33)
\end{abstract}

Keywords: cerebral blood flow; pulmonary hypertension of the newborn; tolazoline; hyperventilation; nitric oxide

Several treatments for severe neonatal respiratory failure have been developed over the past decade. These include high frequency oscillatory ventilation, ${ }^{1}$ surfactant replacement, ${ }^{2}$ and extracorporeal membrane oxygenation (ECMO). ${ }^{3}$ Despite these advances, persistent pulmonary hypertension of the newborn (PPHN) remains a potentially life threatening disease with high morbidity and mortality during the neonatal period. ${ }^{45}$ PPHN is usually treated with tolazoline infusions $(\mathrm{Tz})$ and hyperventilation alkalosis (HAT). Although both treatments have a strong effect on pulmonary vasodilatation, unexpected side effects such as systemic hypotension due to the vasodilatory action on systemic circulation and reduced cerebral blood flow due to excess hypocapnia also occur. ${ }^{6}$ These adverse effects could be related, in part, to the long term neurological sequelae common among survivors of PPHN. On the other hand, inhaled nitric oxide treatment (iNO) has been described as a selective pulmonary dilator in different experimental models and in human infants. ${ }^{8-11}$ iNO has recently been used for the treatment of PPHN in multicentre randomised control trials. ${ }^{12-14}$ Yet, its long term effects on the central nervous system are unknown. The influence of nitric oxide synthases on cerebral arteries and the regulation of cerebral blood flow is widely accepted. ${ }^{15}$ Thus iNO might affect cerebral circulation by altering the activity of nitric oxide synthases or changing systemic circulation. Although it is important to evaluate cerebral blood flow during iNO treatment of PPHN, only two studies using a fetal sheep or newborn lamb model have been reported. ${ }^{16-17}$

Because cerebral circulation is regulated by several factors and this autoregulation changes at different developmental stages during the perinatal period, it is important to investigate the effect of iNO on cerebral circulation of the term neonate, in whom most cases of PPHN occur. Furthermore, the newborn lamb model was induced with bacterial toxin. A different PPHN model is therefore required.

This study investigates mean pulmonary arterial pressure (PAP), mean systemic arterial pressure (SAP), and cerebral blood flow volume (CBF) during treatment for PPHN, using newborn piglets under hypoxic conditions as a model of PPHN. The hypothesis tested whether iNO, compared with other available treatments, induces selective pulmonary vasodilatation without affecting cerebral blood flow in a term newborn model of PPHN.

\section{Methods}

Fifteen newborn piglets between 1 and 3 days of age, mean body weight $2.1 \mathrm{~kg}$, were given 10 $\mathrm{mg} / \mathrm{kg}$ pentobarbital sodium intravenously under ether anaesthesia. After general anaesthesia each newborn piglet underwent tracheostomy and was intubated with an endotracheal tube $(3.0 \mathrm{~mm})$, which was secured in place. After the injection of $0.2 \mathrm{mg} / \mathrm{kg}$ pancuronium animals were ventilated using a pressure limited, time cycled ventilator (BP2001, Bear Medical Systems Inc., USA). At the start of the experiment the respiratory rate was set at 20/min, inspiratory:expiratory ratio at 1:5, PEEP at $0.4 \mathrm{kPa}$, PIP at $1.2 \mathrm{kPa}$, gas flow at 10 $1 / \mathrm{min}$, and $\mathrm{FIO}_{2}$ at 0.3 . The respiratory rate was then adjusted to that of a $\mathrm{PaCO}_{2}$ between 5.3 and $7.3 \mathrm{kPa}$. End tidal $\mathrm{CO}_{2}$ and oxygen saturation were continuously monitored with a pulse oximeter (model 5200, Nellcore-CMI, USA). A right carotid artery catheter was inserted to allow the measurement of SAP and heart rate, using a multichannel amplifier with a thermal recorder (RMP-6018, Nihonkoden, Japan), and an electromagnetic flow transducer (1.5 mm; MVF-3100, Nihonkoden, Japan) was placed in the left carotid artery to measure $\mathrm{CBF}$. Another arterial catheter was placed into 
Table 1 Blood gas, $p H$, base excess, heart rate, PAP, SAP, and cerebral blood flow volume in newborn piglets with PPHN model during treatments

\begin{tabular}{|c|c|c|c|c|c|}
\hline Parameters & Group & Baseline & Before treatment & After treatment & Difference (after-before) \\
\hline \multirow[t]{3}{*}{$\mathrm{PaO}_{2}(\mathrm{kPa})$} & $\mathrm{Tz}$ & $12.5(0.5)$ & $4.0(0.3)^{\star}$ & $4.2(0.4)^{\star}$ & $0.19(0.14)$ \\
\hline & HAT & $11.7(0.7)$ & $4.1(0.3)^{\star}$ & $3.7(0.1)^{\star}$ & $-0.40(0.32)$ \\
\hline & iNO & $12.1(0.4)$ & $4.2(0.2)^{\star}$ & $4.4(0.2)^{\star}$ & $0.21(0.29)$ \\
\hline \multirow{3}{*}{$\mathrm{PaCO}_{2}(\mathrm{kPa})$} & $\mathrm{Tz}$ & $6.3(0.3)$ & $6.1(0.3)$ & $5.9(0.3)$ & $-0.21(0.12)$ \\
\hline & HAT & $6.2(0.1)$ & $6.6(0.3)$ & $3.7(0.1)^{\star \star}$ & $-2.82(0.33)$ \\
\hline & iNO & $6.4(0.2)$ & $6.4(0.2)$ & $6.4(0.2)$ & $-0.03(0.05)$ \\
\hline \multirow[t]{3}{*}{$\mathrm{pH}$} & $\mathrm{Tz}$ & $7.33(0.02)$ & $7.32(0.01)$ & $7.37(0.02)$ & $0.05(0.03)$ \\
\hline & HAT & $7.33(0.01)$ & $7.31(0.03)$ & $7.52(0.01)^{\star \star}$ & $0.21(0.03)$ \\
\hline & iNO & $7.34(0.02)$ & $7.36(0.02)$ & $7.36(0.02)$ & $0.003(0.03)$ \\
\hline \multirow[t]{3}{*}{ Base excess $(\mathrm{mEq} / \mathrm{l})$} & $\mathrm{Tz}$ & $0.1(0.7)$ & $-0.6(1.2)$ & $-0.4(0.2)$ & $0.2(1.4)$ \\
\hline & HAT & $0.4(0.4)$ & $-0.8(1.2)$ & $0.0(1.0)$ & $0.8(0.7)$ \\
\hline & iNO & $0.2(1.0)$ & $1.8(1.5)$ & $1.8(1.5)$ & $0.0(0.5)$ \\
\hline \multirow[t]{3}{*}{ Heart rate (beats/min) } & $\mathrm{Tz}$ & $220(9)$ & $235(10)$ & $222(18)$ & $-14.6(6.4)$ \\
\hline & HAT & $220(13)$ & $234(7)$ & $257(4)$ & $21.6(7.3)$ \\
\hline & iNO & $218(9)$ & $247(9)$ & $245(10)$ & $-1.6(10.5)$ \\
\hline \multirow[t]{3}{*}{ PAP (mm Hg) } & $\mathrm{Tz}$ & $21.7(1.4)$ & $37.9(3.8)^{\star}$ & $27.9(3.3) \dagger$ & $-10.0(1.5)$ \\
\hline & HAT & $21.1(1.2)$ & $41.0(1.8)^{\star}$ & $28.6(1.9) \dagger$ & $-12.4(1.2)$ \\
\hline & iNO & $20.7(1.9)$ & $40.3(2.7)^{\star}$ & $29.5(1.7) \dagger$ & $-10.8(1.6)$ \\
\hline \multirow[t]{3}{*}{$\mathrm{SAP}(\mathrm{mm} \mathrm{Hg})$} & $\mathrm{Tz}$ & $72.6(2.5)$ & $89.6(5.8)$ & $70.5(5.6) \ddagger$ & $-19.1(1.9)$ \\
\hline & HAT & $84.5(2.5)$ & $95.4(7.4)$ & $79.2(4.9)$ & $-16.2(4.7)$ \\
\hline & iNO & $83.1(4.8)$ & $97.4(8.5)$ & $93.7(9.2)$ & $-3.7(3.8)$ \\
\hline \multirow[t]{3}{*}{ Cerebral blood flow $(\mathrm{ml} / \mathrm{min})$} & $\mathrm{Tz}$ & $43.3(2.3)$ & $52.8(1.5)^{\star}$ & $47.3(1.9)$ & $-7.7(0.7)$ \\
\hline & HAT & $37.8(3.8)$ & $49.3(1.4)^{\star}$ & $30.1(1.3)^{\star \star}$ & $-19.2(0.8)$ \\
\hline & $\mathrm{iNO}$ & $36.9(3.3)$ & $52.2(4.5)^{\star}$ & $50.5(4.1)$ & $-1.7(1.3)$ \\
\hline
\end{tabular}

Mean (SEM), $\mathrm{n}=5$.

$\star$ significant difference $(\mathrm{p}<0.01)$ to the baseline levels. ${ }^{\star \star}$ significant difference $(\mathrm{p}<0.01)$ between before and after treatment and between groups. $\dagger$ significant difference $(p<0.01)$ between before and after treatment. $\ddagger$ significant difference $(p<0.05)$ between before and after treatment.

Tz: tolazoline infusion, HAT: hyperventilation alkalosis, iNO: inhaled nitric oxide, PAP: mean pulmonary arterial pressure, SAP: mean systemic arterial pressure.

the femoral artery to obtain blood samples for blood gas analysis (GEM Premier blood gas analyser, Mallinckrodt, USA). A main pulmonary artery catheter (4 Fr, Berman angiographic catheter, Arrow, USA) was inserted from the right jugular vein to measure PAP (RMP-6018, Nihonkoden, Japan). The placement of the catheter was verified by necropsy at the end of the experiment. The rectal temperature was maintained at $37.5^{\circ} \mathrm{C}$ with a heating blanket. Ringer solution was infused at 10 $\mathrm{ml} / \mathrm{kg} / \mathrm{h}$ into a peripheral vein. After stabilisation the $\mathrm{FIO}_{2}$ was reduced to 0.15 to produce a rise in PAP. Once PAP increased 30\% above baseline, the animals were used as a model of PPHN.

TREATMENT OF PPHN

The animals were randomly assigned to treatment with $\mathrm{Tz}, \mathrm{HAT}$, or iNO, and each animal received each treatment once for recording data. Tolazoline (Yamanouchi, Japan), diluted to $1 \mathrm{mg} / \mathrm{ml}$ with saline, at a dose of $1 \mathrm{mg} / \mathrm{kg}$, was administered via an intravenous route for 3 minutes. The dose was determined from standard protocol. ${ }^{6}$ For HAT, FIO ${ }_{2}$ was further reduced to 0.08 , which was immediately followed by an increase in the respiratory cycle at $60 / \mathrm{min}$ to produce respiratory alkalosis, $\mathrm{pH}$ $>7.5$. To maintain the same $\mathrm{PaO}_{2}$ during HAT the $\mathrm{FIO}_{2}$ was determined in experiments performed before this study. Although the $\mathrm{PaO}_{2}$ remained constant, the $\mathrm{PaCO}_{2}$ fell to less than $4 \mathrm{kPa}$ within 15 minutes, and that level of alkalosis was maintained. NO gas, $900 \mathrm{ppm}$ balanced with $\mathrm{N}_{2}$ (Sumitomoseika, Japan), was introduced through a side connector into the inspiratory gas to produce an inhaled concentration of $30 \mathrm{ppm}$. The NO concentration was calibrated using an NO concentration monitor (PAC-NO, Dragel, Germany).
DATA MONITORING

Heart rate, PAP, and SAP were continuously monitored, and CBF was measured before and after the start of each protocol. Once the monitoring values were constant, the data were recorded. Because tolazoline was administered intravenously for 3 minutes, the data were recorded at the beginning and 2 minutes after administration. After the respiratory cycle was increased for HAT, each value changed gradually. Once end tidal $\mathrm{CO}_{2}$ was constant, it was considered stable. This usually occurred between 10 and 15 minutes after the start of hyperventilation. The data were therefore recorded 15 minutes after HAT. In the case of iNO, PAP began to decrease within one minute, and reached a constant level soon after. The data were therefore recorded at the start of iNO and 5 minutes later. The timing was determined in separate experiments before this study. Arterial blood gas was analysed every time data were recorded.

\section{STATISTICAL METHODS}

All statistical analyses on recorded data were performed using StatView (Version 4.5) software (Abacus Concepts, USA). All data are presented as mean (SEM). Analysis of variance (ANOVA) for repeated measurement was used to compare the effects of treatments in each group. For comparison between groups, significant differences were tested by ANOVA at each point. Significant differences were tested with a post hoc test using the Bonferroni/Dunn correction. P values of less than 0.05 were considered significant.

This investigation was approved by the subcommittee for animal research of Osaka City General Hospital. 
Results

Table 1 shows the change in haemodynamics and blood gas analysis with a low fraction of inspired oxygen in each group. During hypoxic breathing, PAP increased significantly in all groups $(p<0.01)$ without any significant differences between groups. SAP, however, increased slightly in all groups (not significant). CBF increased significantly with significant concomitant decrease in $\mathrm{PaO}_{2}$ across all groups $(\mathrm{p}<0.01)$. Heart rate, $\mathrm{PaCO}_{2}, \mathrm{pH}$, and base excess remained constant. There were no significant differences in values between each group. Although SAP was greater than PAP, pulmonary vasoconstriction occurred during hypoxia, and further experiments to measure changes in PAP, SAP, and $\mathrm{CBF}$ during treatment were carried out.

PAP, SAP, CBF and blood gas variables after treatment, and differences evident before and after treatment are also summarised in table 1 . During treatment PAP decreased significantly in all groups $(p<0.01)$. However, no significant difference was observed between each group. SAP decreased significantly in the $\mathrm{Tz}$ group $(p<0.05), C B F$ decreased significantly in the HAT group $(\mathrm{p}<0.01) . \mathrm{PaCO}_{2}$ and $\mathrm{pH}$ also changed significantly in the HAT group $(p<0.01) . \mathrm{PaO}_{2}$ and base excess remained constant in all groups.

\section{Discussion}

iNO is reported to have a selective pulmonary vasodilatory action and no effect on systemic circulation. $^{8-11}$ The long term neurological outcomes of patients with PPHN treated by iNO, however, are not yet clear. Most patients with PPHN have already had asphyxia during the perinatal period, which can damage the central nervous system. Furthermore, hypoxia due to PPHN can worsen the damage to the central nervous system. Under these conditions, the haemodynamic change in cerebral circulation during treatments must be avoided.

Before the use of iNO becomes widespread, its effects on the central nervous system need to be investigated. The effects of iNO on CBF have been reported in fetal sheep and newborn lambs. ${ }^{1617}$ The ability to regulate $\mathrm{CBF}$ in proportion to haemodynamic change is age related. ${ }^{18}$ That is, the change of $\mathrm{CBF}$ at the time when SAP drops or $\mathrm{PaCO}_{2}$ decreases is different for term and premature infants.

Because PPHN is more common in term infants, any changes in $\mathrm{CBF}$ during iNO should be investigated using a neonatal model. Furthermore, pulmonary hypertension in the newborn lamb model was induced with group B streptococci. ${ }^{17}$ The mechanism by which group B streptococci induces pulmonary hypertension is somewhat different from that in PPHN. Group B streptococci infection not only induces pulmonary hypertension but also systemic hypotension. Therefore, the effect of iNO on CBF needs to be investigated using another type of PPHN model. It is also important to compare the properties of iNO with those of other treatments whose long term outcomes are already known.
Our animal model of PPHN was created using a low oxygen tension gas mixture in newborn piglets. The pulmonary artery is very sensitive to hypoxia during the neonatal period; thus PAP can easily be increased. Furthermore, the pulmonary artery is sensitive to vasodilators. Although SAP was greater than PAP, this animal model could be used to test several treatments for PPHN.

Tz significantly reduced PAP. This, however, does not necessarily increase the effectiveness of the treatment. Tz also greatly reduced SAP; the ratio of the reduction of PAP to the reduction of SAP was almost equal. It has been reported that the use of a vasodilator cannot reduce the mortality of PPHN. ${ }^{19}$ Although Tz did not significantly diminish CBF, the sudden drop in systemic blood pressure might reduce CBF. CBF is usually constant even when the systemic blood circulation changes within a certain range because of an autoregulatory mechanism. This function is thought to be incomplete and easily impaired during the neonatal period. ${ }^{20}$ Therefore if the change in blood pressure is profound and rapid during treatment of PPHN, the CBF may also change. Because $\mathrm{Tz}$ is still a popular drug in many hospitals, every effort to maintain the systemic circulation must be made before administering the drug; this includes the infusion of vasoconstrictive drugs, such as dopamine, and a volume expander. This will help to avoid potential side effects such as systemic hypotension followed by the reduction of CBF.

HAT significantly reduced PAP, while not changing SAP. The reduction in PAP was the greatest achieved by all the treatments studied. Carbon dioxide tension of the artery greatly affects CBF. Hypocarbia during HAT can reduce $\mathrm{CBF}$ by as much as $50 \%{ }^{21}$ In our study HAT had a significant effect on CBF. After the initiation of HAT, CBF was reduced by $40 \%$. Because CBF is regulated by many means, factors other than hypocapnia must be excluded in the experiment. As shown in table $1, \mathrm{PaO}_{2}$ did not change during HAT because of a lowered $\mathrm{FIO}_{2}$. Thus the change in $\mathrm{CBF}$ could not have been created by a change in $\mathrm{PaO}_{2}$.

Another factor possibly affecting $\mathrm{CBF}$ was an increase in MAP. After the start of HAT mean airway pressure increased from $0.4 \mathrm{kPa}$ to $0.8 \mathrm{kPa}$. This increase induced a decrease in $\mathrm{SAP}$ and/or an increase in venous pressure and so could have affected $\mathrm{CBF}$. In a different experiment we studied the effect of increased mean airway pressure on the CBF by increasing PEEP without changing respiratory rate. In this study $\mathrm{PaCO}_{2}$ remained unchanged, so we were able to evaluate the effect of mean airway pressure on CBF. The results showed less than a $10 \%$ reduction in CBF. Thus we can speculate that the huge decrease in CBF during HAT was due mainly to hypocapnia induced by HAT. Of course, we expected a reduction in CBF during HAT, but not by such a large amount (as much as $40 \%$ ). The levels of $\mathrm{PaCO}_{2}$ below $4 \mathrm{kPa}$ could be induced in experimental animals. Furthermore, an asphyxiated newborn infant is probably much less sensitive to a change in $\mathrm{PaCO}_{2}$ in 
regulating $\mathrm{CBF}^{22}$ The changes observed in this study, however, were not small. This reduction explains the adverse outcome of patients with PPHN who are treated with HAT. HAT should only be permitted for a short duration and, as the pulmonary artery is basically sensitive to arterial $\mathrm{pH}$, the $\mathrm{pH}$ should be maintained as high as possible with an alkaline solution.

iNO treatment of PPHN began only recently and has spread rapidly. ${ }^{10} 11$ In the present study, the selective vasodilatation of iNO was shown using a newborn piglet model. This result is consistent with previous reports of different animal models. ${ }^{89}$ Although the reduction in PAP was not the greatest among the treatments studied, the ratio of the reduction of PAP to SAP was the smallest. Thus the actions of iNO in PPHN might be ideal for the resolution of pulmonary hypertension, as indicated in the animal model. No effect on cerebral blood flow during iNO was observed. This result is consistent with that in the study in which fetal sheep or newborn lamb were used. ${ }^{16}{ }^{17}$ Because the animal models in our study were term newborn piglets and were induced with hypoxia, the results are more accurately extrapolated to human term neonates. These results, however, were observed as an acute phase change in cerebral circulation. Thus the long term effects of iNO on $\mathrm{CBF}$ must be examined.

In summary, $\mathrm{Tz}$ had a strong effect on PAP with a strong reduction in SAP. HAT had a strong effect on pulmonary blood pressure with a strong reduction in $\mathrm{CBF}$. On the other hand, iNO strongly reduced PAP without any changes in SAP and CBF. Because the reduction in $\mathrm{CBF}$ during treatment of $\mathrm{PPHN}$ is considered a major cause of subsequent brain damage, iNO is an ideal treatment for PPHN. Thus we would expect a better neurological outcome with iNO treatment than with the conventional treatments, $\mathrm{Tz}$ and HAT. Several control trials have demonstrated the efficacy of iNO for PPHN. ${ }^{12-14}$ Although the long term effects of iNO on newborns are unknown, iNO is a promising treatment for PPHN and could significantly reduce the mortality and morbidity associated with it.
1 Clark RH. High-frequency ventilation. $f$ Pediatr 1994;124:661-70

2 Fuiiwara T Surfactant replacement in neonatal RDS. In: Robertson B, Van Golde LMG, Batenburg JJ eds. Pulmonary Surfactant. Amsterdam: Elsevier, 1984: 479503

3 Short BL, Miller MK, Anderson KD. Extracorporeal membrane oxygenation in the management of respiratory failure in the newborn. Clin Perinatol 1987;14:737-48.

4 Roberts JD, Shaul PW. Advances in the treatment of persistent pulmonary hypertension of the newborn. Pediatr Clin North Am 1983;40:983-1004.

5 Kinsella JP, Abman SH. Recent developments in the pathophysiology and treatment of persistent pulmonary hypertension of the newborn. $\mathcal{F}$ Pediatr 1995;126:853-64.

6 Ward RM. Pharmacology of tolazoline. Clin Perinatol 1984;11:703-13.

7 Bifano EM, Pfannenstiel A. Duration of hyperventilation and outcome in infants with persistent pulmonary and outcome in infants with persis

8 Fratacci MD, Frostell CG, Chen TY, Wain JC, Robinson DR, Zapol WM. Inhaled nitric oxide- a selective pulmonary vasodilator of heparin-protamine vasoconstriction in sheep. Anesthesiology 1991;75:990-9.

9 Frostell C, Fratacci MD, Wain JC, Jones R, Zapol WM. Inhaled nitric oxide-a selective pulmonary vasodilator reversing hypoxic pulmonary vasoconstriction. Circulation 1991;83:2038-47.

10 Roberts JD, Polaner DM, Lang P, Zapol WM. Inhaled nitric oxide in persistent pulmonary hypertension of the newborn. Lancet 1992;340:818-9.

11 Kinsella JP, Neish SR, Shaffer E, Abman SH. Low-dose inhalational nitric oxide in persistent pulmonary hypertension of the newborn. Lancet 1992;340:819-20.

12 The Neonatal Inhaled Nitric Oxide Study Group. Inhaled nitric oxide in full-term and nearly full-term infants with nitric oxide in full-term and nearly full-term infants with
hypoxic respiratory failure. $N$ Engl $\mathcal{F}$ Med 1997;336:597hypoxic 604 .

13 Roberts JD, Fineman JR, Morin FC, et al. Inhaled nitric oxide and persistent pulmonary hypertension of the newoxide and persistent pulmonary hypert
born. $N$ Engl f Med 1997;336:605-10.

14 Kinsella JP, Truog WE, Walsh WF, et al. Randomized, multicenter trial of inhaled nitric oxide and high-frequency oscillatory ventilation in severe, persistent pulmonary hypertension of the newborn. F Pediatr 1997;131:55-62.

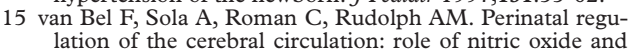
prostaglandins. Pediatr Res 1997;42:299-304

16 Rosenberg AA, Kinsella JP, Abman SH. Cerebral hemodynamics and distribution of left ventricular output during
inhalation of nitric oxide. Crit Care Med 1995;26:1391-7.

17 Lopes Cardozo RH, de Beaufort AJ, Gesink BJ, et al. Inhalation of nitric oxide: effect on cerebral hemodynamic and activity, and antioxidant status in the newborn lamb. Biol Neonate 1996;69:284-92.

18 Haaland K, Karlsson B, Skovlund E, Lagercrantz H, Thoresen M. Postnatal development of the cerebral blood flow velocity response to changes in $\mathrm{CO}_{2}$ and mean arterial blood pressure in the piglets. Acta Paediatr 1995;84:141420.

19 Stevenson DK, Kasting DS, Darnall RA, et al. Refractory hypoxemia associated with neonatal pulmonary disease: The use and limitation of tolazoline. $\mathcal{f}$ Pediatr 1979;95:595-9.

20 Younkin DP, Reivich M, Jaggi JL, Obrist WD, DelivoriaPapadopoulos $M$. The effect of hematocrit and systolic blood pressure on cerebral blood flow in newborn infants. f Cereb Blood Flow Metab 1987;7:295-9.

21 Gleason CA, Short BL, Jones MD. Cerebral blood flow and metabolism during and after prolonged hypocapnia in newborn lambs. F Pediatr 1989;115:309-14.

22 Greisen G. Cerebral blood flow and energy metabolism in the newborn. Clin Perinatol 1997;24:531-46. 